\title{
UNDERSTANDING THE $f_{0}(980)$ AND $a_{0}(980)$ MASSES AS WELL AS THEIR WIDTHS
}

\author{
E. VAN BEVEREN \\ Centro de Física da UC \\ Departamento de Física, Universidade de Coimbra \\ P-3004-516 Coimbra, Portugal \\ E-mail: eef@uc.pt \\ G. RUPP \\ Centro de Física e Engenharia de Materiais Avançados \\ Instituto Superior Técnico, Universidade de Lisboa \\ P-1049-001 Lisbon, Portugal \\ E-mail: george@ist.utl.pt
}

\begin{abstract}
The low and approximately equal masses of the scalar mesons $f_{0}(980)$ and $a_{0}(980)$, as well as their relatively small decay widths, are impossible to understand in terms of standard $P$-wave quark-antiquark states. Here, these mesons are studied in a unitarised quark-meson model, together with the other light isoscalar scalar $f_{0}(500)$, as members of a complete scalar nonet below about $1 \mathrm{GeV}$. They are shown to be dynamical states generated by a combination of quark-confinement and strong-decay interactions, resulting in a large breaking of $S U(3)_{\text {flavour }}$ symmetry. This is illustrated with several pole trajectories in the complex-energy plane as a function of the model's decay coupling constant.

Also, experimental evidence is presented of a still much lighter scalar boson called $E(38)$, which may correspond to a novel kind of mesons predicted by V. N. Gribov, as an observable manifestation of a condensate of light quarks.
\end{abstract}

\section{Introduction: light scalar-meson nonet}

The ground-breaking work of V. N. Gribov on quark confinement, published after his death in two edited papers, ${ }^{1}$ suggested the possibility of a condensate of light quark pairs and the consequent existence of a new kind of mesons that would manifest a very strong breaking of $S U(3)_{\text {flavour }}$ symmetry. In those days, the early 1990s, there were indeed two scalar mesons in the tables of the Particle Data Group whose properties seemed to rule them out as standard quark-model mesons, viz. the almost massdegenerate and relatively narrow $f_{0}(975)$ and $a_{0}(980)$ resonances. ${ }^{2}$ Thus, 
Gribov and co-authors argued ${ }^{3}$ that these two scalars could be examples of such "novel" mesons. Their quark-antiquark configurations would then be $(u \bar{u}+d \bar{d}) / \sqrt{2}$ for the isoscalar $f_{0}(975)$ and $(u \bar{u}-d \bar{d}) / \sqrt{2}$ for the isovector $a_{0}(980)$, under the assumption that here the $u, \bar{u}$ and $d, \bar{d}$ flavours carry negative kinetic energy.

However, historically the status of the light scalar mesons had been even more complicated, with the additional very broad isoscalar $\epsilon(700)$ and isodoublet $\kappa(725)$ included in the PDG tables as early as the late 1960 s (see minireview ${ }^{4}$ ). The corresponding highly unusual pattern of scalar-meson masses and widths led R. L. Jaffe ${ }^{5}$ to propose tetraquark assignments for a complete light scalar nonet, which would e.g. naturally explain the approximate mass degeneracy of $f_{0}(975)$ and $a_{0}(980)$, based on an equal four-quark content. Nevertheless, already a year earlier the PDG had dropped the entries of the light $\epsilon$ and $\kappa$ resonances in the tables, replacing ${ }^{4}$ them by states well above $1 \mathrm{GeV}$. This situation was maintained till 1996, with the reintroduction of a light $\epsilon$ in the PDG tables, now called $f_{0}$ under the new naming scheme and with a huge mass range of $400-1200 \mathrm{MeV}$ in order to accommodate several conflicting analyses. ${ }^{4}$ Finally, the PDG recovered a light $\kappa$ entry in the 2004 tables, with the new name $K_{0}^{\star}(800)$. Very detailed data analyses in recent years gave rise to the further renamings $f_{0}(600)$ (2002), $f_{0}(500)(2012)$, and $K_{0}^{\star}(700)$ (2018), besides the change to $f_{0}(980)$ instead of $f_{0}(975)$ already in 1994 . Together with $a_{0}(980)$, these scalar mesons constitute a complete light nonet in the current PDG tables. ${ }^{6}$

In the present paper, a unitarised quark model will be revisited that long ago predicted ${ }^{7}$ such a light scalar-meson nonet, namely as additional and dynamical $q \bar{q}$ states owing their existence to an interplay between the confinement and strong-decay mechanisms (see e.g. Ref. ${ }^{8}$ for very recent work on this topic). Manifest $S$-matrix unitarity and analyticity were crucial for the model's predictions of scalar-meson masses and widths that are still today fully compatible with PDG limits. ${ }^{6}$ The original model was developed in coordinate space, but here we shall discuss ${ }^{9}$ a more recent, momentum-space formulation, ${ }^{10}$ which has the advantage of being both algebraically and analytically solvable, besides dealing more appropriately with non-localities. Trajectories of $S$-matrix poles will be shown to demonstrate the uncommon behaviour of these "novel" mesons. Nevertheless, they should be understood as normal positive-energy phenomena resulting from the very strong coupling between confined $P$-wave $q \bar{q}$ states and free $S$-wave two-meson states.

As an addendum, strong experimental indications of a very light new 
boson, recently observed ${ }^{11}$ at the Dubna JINR and predicted ${ }^{12,13}$ by us almost a decade ago, will be shown and discussed. This tentative $E(38)$ boson may indeed be one of the novel mesons foreseen by Gribov.

The organisation of this paper is as follows. In Sec. 2 we very briefly review our unitarised quark-meson model as it was originally formulated in coordinate space. In Sec. 3 the corresponding momentum-space version is recapitulated and results of fits to the data are presented. Section 4 deals with the tentative $E(38)$ boson. In Sec. 5 we draw some conclusions and suggest possible future work. For a very recent general review on the crucial role of unitarity in meson spectroscopy, see Ref. ${ }^{14}$.

\section{Unitarised quark-meson model in coordinate space}

A non-relativistic unitarised quark-meson model, developed in the late 1970s at the University of Nijmegen, allowed a very good reproduction of the then known vector charmonium and bottomonium spectra. ${ }^{15}$. It was based on harmonic-oscillator (HO) confinement with a universal frequency $\omega$ and the ${ }^{3} P_{0}$ model for strong decay, the latter represented by a spherical delta-shell to mimic the transitions between the confined heavy $q \bar{q}$ sector and the free open-charm or open-bottom channels. However, the model produced too small hadronic widths, despite the generated large real mass shifts as a consequence of unitarisation.

Therefore, a generalisation of the model was formulated ${ }^{16}$ with a smeared-out transition potential, besides the employment of relativistic kinematics in the open two-meson channels in order allow application to mesons in an extended mass range, in particular those with pions in their decay modes. Thus, all two-meson decay channels consisting of combinations of ground-state pseudoscalar and vector mesons allowed by quantum numbers were included. The equations were solved analytically through an approximation of the smooth decay potential by an increasing number of delta-shells until convergence with a precision of a few $\mathrm{MeVs}$ was achieved. Further phenomenological ingredients were colour hyperfine splitting from one-gluon exchange and an energy-dependent suppression of kinematically closed two-meson channels. Fits were then carried out to light, heavy-light, and heavy vector as well as pseudoscalar mesons, with globally good results for the vectors and reasonable ones for the pseudoscalars, especially considering the small number of parameters ( 8 in total) and the large variety of described mesons. The parameters were the HO frequency $\omega=190 \mathrm{MeV}$, a dimensionless coupling for ${ }^{3} P_{0}$ decay $g^{2} / 4 \pi=7.59$, a dimensionless overall 
coupling for colour splitting $g_{c}=5.47$, a dimensionless peak radius of the ${ }^{3} P_{0}$ decay potential $\rho_{0}=0.56$, and the four constituent quark masses

$$
m_{n}=406 \mathrm{MeV}, m_{s}=508 \mathrm{MeV}, m_{c}=1562 \mathrm{MeV}, m_{b}=4724 \mathrm{MeV} \text {, }
$$

where $m_{n} \equiv m_{u}=m_{d}$. Note that in this model hadronic widths were not computed perturbatively and a posteriori, but both masses and widths followed directly from complex resonance pole positions in a manifestly unitary multichannel $S$-matrix. Thus, even overlapping broad resonances would still automatically satisfy unitarity.

These features made the model ideal to study the very controversial light scalar mesons, in view of the very unusual pattern of scalar masses and widths and even their questionable existence as true quark-model resonances. Thus, in Ref. ${ }^{7}$ a complete light scalar-meson nonet was found, as additional and dynamical resonances calculated in the above multichannel model ${ }^{16}$ with completely unaltered parameters, Now, 34 years later, those predictions for pole positions (in MeV), viz. $\epsilon(470-i 208)\left(f_{0}(500)\right)$, $S^{\star}(994-i 20)\left(f_{0}(980)\right), \kappa(727-i 263)\left(K_{0}^{\star}(700)\right)$, and $\delta(968-i 28)\left(a_{0}(980)\right)$ are still fully within the latest $\mathrm{PDG}^{6}$ limits. In Fig. 1 we also show the computed $S$-wave $\pi \pi$ phase shift, obtained without any fit, together with the then available data (see Ref. ${ }^{7}$ ). Besides the mentioned light scalar mesons, the model also predicted another complete light scalar nonet at roughly 1.3$1.5 \mathrm{GeV}$, corresponding to a few then already observed ${ }^{17} 0^{+(+)}$resonances and also in agreement with expectations from the static quark model for

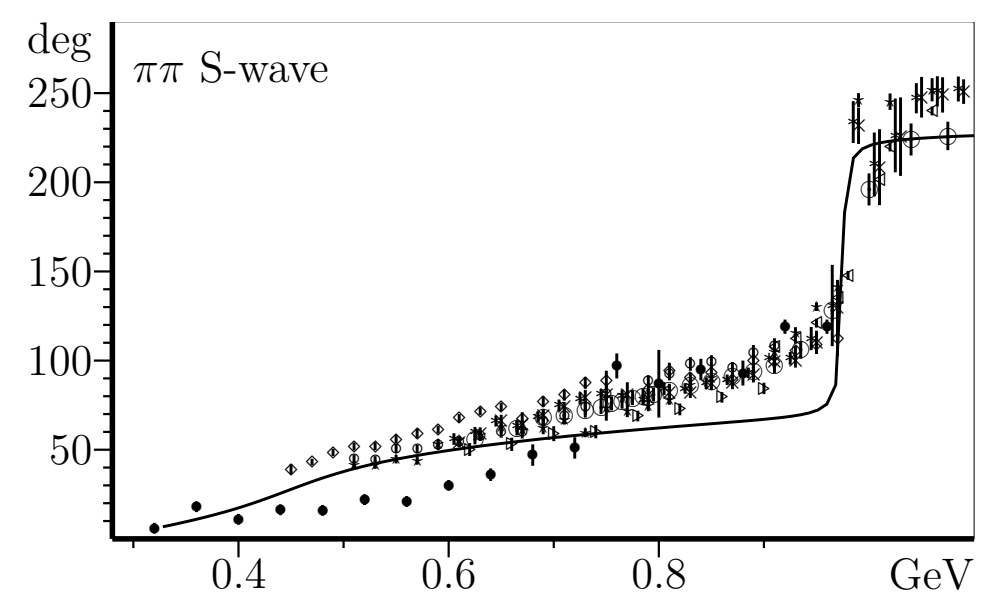

Figure 1. $S$-wave $\pi \pi$ phases predicted in $\operatorname{Ref}^{7}{ }^{7}$ (see this reference for data). 
standard $P$-wave $q \bar{q}$ states.

\section{Scalar mesons in a unitarised momentum-space model}

The coordinate-space models presented in the previous section had achieved a remarkable good description of the whole meson spectrum, in particular the light scalar mesons. It allowed for the first time to reproduce these controversial resonances as a complete scalar nonet in the context of the quark model, yet not as regular quark-antiquark mesons but instead as dynamical complex-energy poles that cannot be linked in a simple way to the discrete confinement spectrum. However, especially the more general model of Ref. ${ }^{16}$ was based on a very complicated derivation of the multichannel $S$-matrix and so not very accessible to experimentalists in their quest for unitary multichannel tools to analyse the spectroscopic data.

Thus, we first developed ${ }^{18}$ a simple yet non-perturbative momentumspace version of the model, which in the limit of a small decay constant gives rise to Breit-Wigner-like expressions for resonances. A detailed derivation of this so-called Resonance-Spectrum Expansion (RSE), employing like in Ref. ${ }^{15}$ a single spherical delta-shell in coordinate space to mimic the ${ }^{3} P_{0}$ decay potential, can be found in Ref. ${ }^{10}$. The RSE model, for one confined quark-antiquark channel coupled to one free meson-meson (MM) channel, was applied in Ref. ${ }^{18}$ to show that the then still unlisted ${ }^{19}$ scalar $\kappa$ resonance could be easily extracted from the available experimental data on $S$-wave $K \pi$ scattering, with a pole position that is compatible with the present PDG limits for the $K_{0}^{\star}(700)$ resonance. ${ }^{6}$ Exactly the same model was the first successful description ${ }^{20}$ of the then newly discovered and very enigmatic scalar charm-strange meson $D_{s 0}^{\star}(2317),{ }^{6}$ the only change being the replacement of a light-quark mass by a charm-quark mass.

Now, this simple model was adequate to describe $K_{0}^{\star}(700)$ and $D_{s 0}^{\star}(2317)$, because both resonances couple to only one quark-antiquark state and all but the lowest MM channel that couple lie at much higher energies. However, in general there are more relevant decay modes and in many cases also more than one quark-antiquark channel, e.g. in the case of vector mesons, where there are both ${ }^{3} S_{1}$ and ${ }^{3} D_{1} q \bar{q}$ components. The first generalisation was presented and used in Ref. ${ }^{21}$, namely to model the light scalar mesons with all pseudoscalar-pseudoscalar (PP) decay channels included and also both $n \bar{n} \equiv(u \bar{u}+d \bar{d}) / \sqrt{2}$ and $s \bar{s}$ quark-antiquark channels in the isosinglet case. This multichannel model is also manifestly unitary, but only when the dependence of the channel couplings on the radial quan- 
tum number $n$ (see below) is the same for all considered MM channels, which is the case for $S$-wave PP channels. A further generalisation ${ }^{9}$ was therefore due in order to cover all possible $n$ dependencies, which is the model employed in the present study.

The momentum-space model allows a very simple and instructive diagrammatic representation of the $T$-matrix for MM scattering with nonexotic quantum numbers, viz. $^{9}$

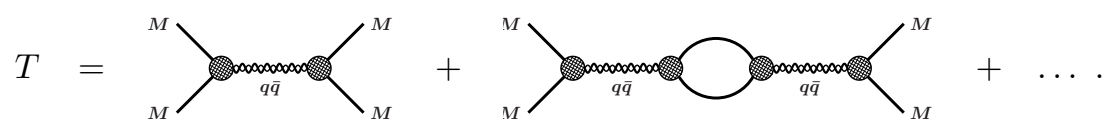

Here, the first diagam on the right-hand side stands for the effective MM potential $V$ generated by the $\operatorname{MM} q \bar{q}$ vertices and the $q \bar{q}$ propagator in the intermediate state. The wiggly lines indicate that this is not just one $q \bar{q}$ state but a whole, in principle infinite tower of $q \bar{q}$ states with the same quantum numbers, and therefore a kind of Regge propagator. ${ }^{22}$ The second diagram is the once-iterated $V$, with an MM loop in between, and so the second term in a Born series. Since $V$ is separable, as it is only based on $s$ channel exchanges, the whole series can be summed up easily, giving rise to a closed-form expression for the $T$-matrix. The explicit momentum-space formulae for $V \operatorname{read}^{23}$

$$
\begin{gathered}
V_{i j}^{L_{i}, L_{j}}\left(p_{i}, p_{j}^{\prime} ; E\right)=\lambda^{2} j_{L_{i}}^{i}\left(p_{i} a\right) \mathcal{R}_{i j}(E) j_{L_{j}}^{j}\left(p_{j}^{\prime} a\right), \\
\mathcal{R}_{i j}(E)=\sum_{i_{q \bar{q}}=1}^{N_{q \bar{q}}} \sum_{n=0}^{\infty} \frac{g_{\left(i_{q \bar{q}}, n\right)}^{i} g_{\left(i_{q \bar{q}}, n\right)}^{j}}{E-E_{n}^{\left(i_{q \bar{q}}\right)}}
\end{gathered}
$$

where the RSE propagator $\mathcal{R}$ contains an infinite tower of $s$-channel bare $q \bar{q}$ states, corresponding to the discrete spectrum of an arbitrary confining potential. Here, $E_{n}^{\left(i_{q \bar{q}}\right)}$ is the energy level of the $n$-th recurrence in the $i_{q \bar{q}}$-th $q \bar{q}$ channel, with $N_{q \bar{q}}$ the number of $q \bar{q}$ channels having the same quantum numbers, and $g_{\left(i_{q \bar{q}}, n\right)}^{i}$ is the corresponding coupling to the $i$-th MM channel. Furthermore, in Eq. (2), $\lambda$ is the overall coupling constant for ${ }^{3} P_{0}$ decay, and $j_{L_{i}}^{i}\left(p_{i}\right)$ and $p_{i}$ are the $L_{i}$-th order spherical Bessel function and the (relativistically defined) off-shell relative momentum in MM channel $i$, respectively. The spherical Bessel function originates in our string-breaking picture of OZI-allowed decay, being just the Fourier transform of a spherical delta-shell of radius $a$. The channel couplings $g_{\left(i_{q \bar{q}}, n\right)}^{i}$ in Eq. (3) are computed following the formalism developed in Ref. ${ }^{24}$, namely from overlaps of HO wave functions for the original $q \bar{q}$ pair, the created ${ }^{3} P_{0}$ pair, and the 
quark-antiquark states corresponding to the outgoing two mesons. In most cases, this method produces the same couplings for ground-state mesons as the usual point-particle approaches, but also provides a clear prescription for excited mesons, with the additional advantage of always resulting in a finite number of non-vanishing couplings. Because of their fast decrease for increasing radial quantum number $n$, practical convergence of the infinite sum in Eq. (3) is achieved by truncating it after at most 20 terms.

With this effective energy-dependent MM potential, the $T$-matrix reads explicitly $^{23}$

$$
\begin{gathered}
T_{i j}^{\left(L_{i}, L_{j}\right)}\left(p_{i}, p_{j}^{\prime} ; E\right)=-2 a \lambda^{2} \sqrt{\mu_{i} p_{i}} j_{L_{i}}^{i}\left(p_{i} a\right) \times \\
\sum_{m=1}^{N} \mathcal{R}_{i m}\left\{[\mathbb{1}-\Omega \mathcal{R}]^{-1}\right\}_{m j} j_{L_{j}}^{j}\left(p_{j}^{\prime} a\right) \sqrt{\mu_{j} p_{j}^{\prime}}
\end{gathered}
$$

with the loop function

$$
\Omega_{i j}\left(k_{j}\right)=-2 i a \lambda^{2} \mu_{j} k_{j} j_{L_{j}}^{j}\left(k_{j} a\right) h_{L_{j}}^{(1) j}\left(k_{j} a\right) \delta_{i j},
$$

where $h_{L_{j}}^{(1) j}\left(k_{j} a\right)$ is the spherical Hankel function of the first kind, $k_{j}$ and $\mu_{j}$ are the on-shell relative momentum and reduced mass in MM channel $j$, respectively, and the matrix $\mathcal{R}(E)$ is given by Eq. (3). Note that no regularisation is needed in this all-orders model, since the Bessel functions at the vertices make the meson loops finite.

Now we are in a position to apply this model to scalar mesons just like in Ref. ${ }^{9}$. However, since here we focus on the scalars $f_{0}(980)$ and $a_{0}(980)$, we do not deal with $K_{0}^{\star}(700)$ and only consider the mixed isoscalars $f_{0}(500) / f_{0}(980)$ and the isovector $a_{0}(980)$. The following PP, vector-vector $(\mathrm{VV})$, and scalar-scalar (SS) two-meson decay channels are included:

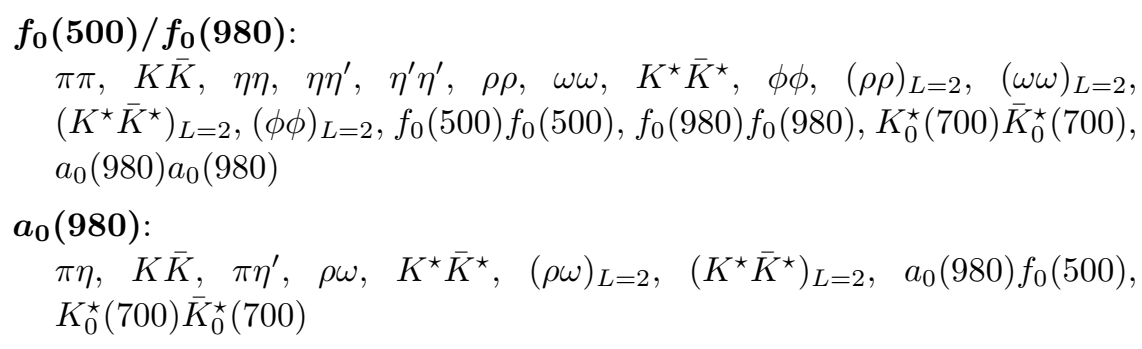

Note that all channels are in $S$-waves, except for those marked with $L=2$. It may also be considered strange that some decay channels are included with the same mesons as the ones that we are going to describe, which looks like a (partial) bootstrap. However, by including these channels we try to mimic e.g. the observed ${ }^{6}$ decay modes $f_{0}(1370) \rightarrow 2(\pi \pi)_{S \text {-wave }}$ and 
$a_{0}(1450) \rightarrow a_{0}(980) \pi \pi$, in which the two-pion final states are probably dominated by the $f_{0}(500)$ resonance. As for the confined sector, we only need the $(u \bar{u}-d \bar{d}) / \sqrt{2}$ channel in the isovector case, However, for isoscalars $n \bar{n}=(u \bar{u}+d \bar{d}) / \sqrt{2}$ and $s \bar{s}$ can mix and couple to both $f_{0}(500)$ and $f_{0}(980)$. Part of this mixing will inevitably proceed via $K \bar{K}$ loops, but some mixing purely on the quark level should also be considered. A strong indication comes from the relatively large hadronic decay width of the scalar charmonium state $\chi_{c 0}(1 P)$, viz. about $10 \mathrm{MeV},{ }^{6}$ in spite of being due to OZI-forbidden processes only. For comparison, the total width of the vector charmonium state $J / \psi$ is only $0.093 \mathrm{MeV}$. This difference is usually attributed to $C$-parity, which allows hadronic decays of a $J^{P C}=0^{++}$state via $q \bar{q}$ annihilation to two intermediate gluons, whereas for a $J^{P C}=1^{--}$ state three gluons are needed to conserve $C$-parity. Thus, in our coupled $f_{0}(500) / f_{0}(980)$ case, we take two $q \bar{q}$ channels that are already mixtures of pure $n \bar{n}$ and $s \bar{s}$ states, with bare ground-state masses $M_{1}$ and $M_{2}$ given by the quadratic mass formulae $e^{25}$

$$
\begin{aligned}
& M_{n \bar{n}}^{2}=\left(M_{1} \cos \Theta_{\mathrm{S}}\right)^{2}+\left(M_{2} \sin \Theta_{\mathrm{S}}\right)^{2}, \\
& M_{s \bar{s}}^{2}=\left(M_{1} \sin \Theta_{\mathrm{S}}\right)^{2}+\left(M_{2} \cos \Theta_{\mathrm{S}}\right)^{2},
\end{aligned}
$$

where $\Theta_{\mathrm{S}}$ is the scalar mixing angle, to be fitted next. In the isovector case, one $(u \bar{u}-d \bar{d}) / \sqrt{2}$ channel is naturally sufficient. In both cases, we take the bare RSE energy levels to be given by an HO spectrum with constant frequency $\omega$, like e.g. in Refs. ${ }^{21,9}$, i.e.,

$$
E_{\mathrm{HO}}=(2 n+\ell+3 / 2) \omega+m_{q_{1}}+m_{q_{2}},
$$

with $n$ and $\ell$ the radial and orbital quantum numbers, respectively, and $m_{q_{1}}, m_{q_{2}}$ the constituent quark masses. So $m_{q_{1}}+m_{q_{2}}$ is taken as $M_{1}$ from Eqs. $(6,7)$ for $f_{0}(500)$ and its higher recurrences, as $M_{2}$ for $f_{0}(980)$, and as $M_{n \bar{n}}=2 m_{n}$ for $a_{0}(980)$. A final phenomenological ingredient of our present model is an additional subthreshold suppression of kinematically closed MM channels, as the loop function in Eq. (5) turns out to be insufficient for that purpose in the fits to the data. Such a suppression was dealt with in previous work by multiplying the overall coupling $\lambda$ with a factor $E / T_{i}$ (see e.g. Ref. ${ }^{16}$ ), with $T_{i}$ the threshold mass of the relevant channel, ${ }^{16}$ or by a form factor $\exp \left(-\alpha\left|k_{i}\right|^{2}\right.$ ) (see e.g. Ref. ${ }^{21}$ ), where $k_{i}$ is the channel's relative momentum and $\alpha$ an adjustable constant. Here, we opt for the latter solution but written analytically as $\exp \left(\alpha k_{i}^{2}\right)$, which allows a more rigorous continuation into the complex-energy plane when searching for 
poles (see below). This damping constant is taken as $\alpha=6 \mathrm{GeV}^{-2}$ in the isoscalar case and $\alpha=3 \mathrm{GeV}^{-2}$ for the isovector.

The few free parameters of the model are fitted to $S$-wave $\pi \pi$ phase shifts from threshold up to $1.6 \mathrm{GeV}$ in the isoscalar case and to the $a_{0}(980)$ line shape for the isovector (see Ref. ${ }^{21}$ for the used data). For stability reasons, we do not fit $\alpha$, but select its optimum value by hand in either case. Note that we take the values of $m_{n}, m_{s}$, and $\omega$ unchanged as in all our previous work, with $\omega=190 \mathrm{MeV}$ and $m_{n}, m_{s}$ given in Eq. (1). Thus, the isoscalar fit parameters are the coupling constant $\lambda$, the decay radius $a$, and the scalar and pseudoscalar mixing angles $\Theta_{\mathrm{S}}$ and $\Theta_{\mathrm{PS}}$, the latter being needed to properly determine the couplings to channels involving the $\eta$ and/or $\eta^{\prime}$ mesons. In the isovector case, the free parameters are $\lambda, \Theta_{\mathrm{PS}}$, $a_{n \bar{n}}$ and $a_{s \bar{s}}$, where the latter two decay radii are taken different for $n \bar{n}$ and $s \bar{s}$ pair creation. For only one $q \bar{q}$ channel, this is possible without changing the structure of Eqs. $(2-5)$, which is not feasible when there are more than one $q \bar{q}$ channels. The fitted parameters are:

$f_{0}(500) / f_{0}(\mathbf{9 8 0})$ :

$\lambda=3.557 \mathrm{GeV}^{-1 / 2}, a=3.417 \mathrm{GeV}^{-1}, \Theta_{\mathrm{S}}=17.54^{\circ}, \Theta_{\mathrm{PS}}=41.78^{\circ}$

$a_{0}(980)$ :

$\lambda=2.132 \mathrm{GeV}^{-1 / 2}, a_{n \bar{n}}=3.944 \mathrm{GeV}^{-1}, a_{s \bar{s}}=2.900 \mathrm{GeV}^{-1}, \Theta_{\mathrm{PS}}=42.33^{\circ}$

First of all, it is very reassuring that in both fits $\Theta_{\mathrm{PS}}$ comes out close to $42^{\circ}$, which is the value favoured in many experimental and theoretical analyses. ${ }^{25}$ Moreover, also $\Theta_{\mathrm{S}}$ is near what one would expect from estimates $^{25}$ based on quadratic mass formulae and certain decay rates, namely roughly $20^{\circ}$. Finally, $a$ comes out right in the middle of $a_{n \bar{n}}$ and $a_{s \bar{s}}$, which lends additional support to the physical significance of the fits. The fact that $\lambda$ comes out considerably larger in the isoscalar case than for the isovector may due be to a deficient handling of the important $4 \pi$ modes $^{6}$ above roughly $1 \mathrm{GeV}$. This is also indicated by the fitted phase shifts, which reasonably reproduce the experimental ones up to about the $K \bar{K}$ threshold, but thereabove first show an undershoot followed by an overshoot. We shall come back to this point in Sec. 5. As for the fitted line shape of $a_{0}(980)$, we get deviations of less than $2 \%$ from $900 \mathrm{MeV}$ all the way up to near the $\pi \eta^{\prime}$ threshold at $1093 \mathrm{MeV}$. It lies outside the scope of the present paper to show details of the fits, since the main issue here is the nature of the $f_{0}(980)$ and $a_{0}(980)$ resonances. For the fitted parameters, we find the following most important poles (in $\mathrm{MeV}$ ):

$f_{0}(455-i 232), f_{0}(1007-i 17.4)$ (second sheet), $f_{0}(1290-i 131)$ 

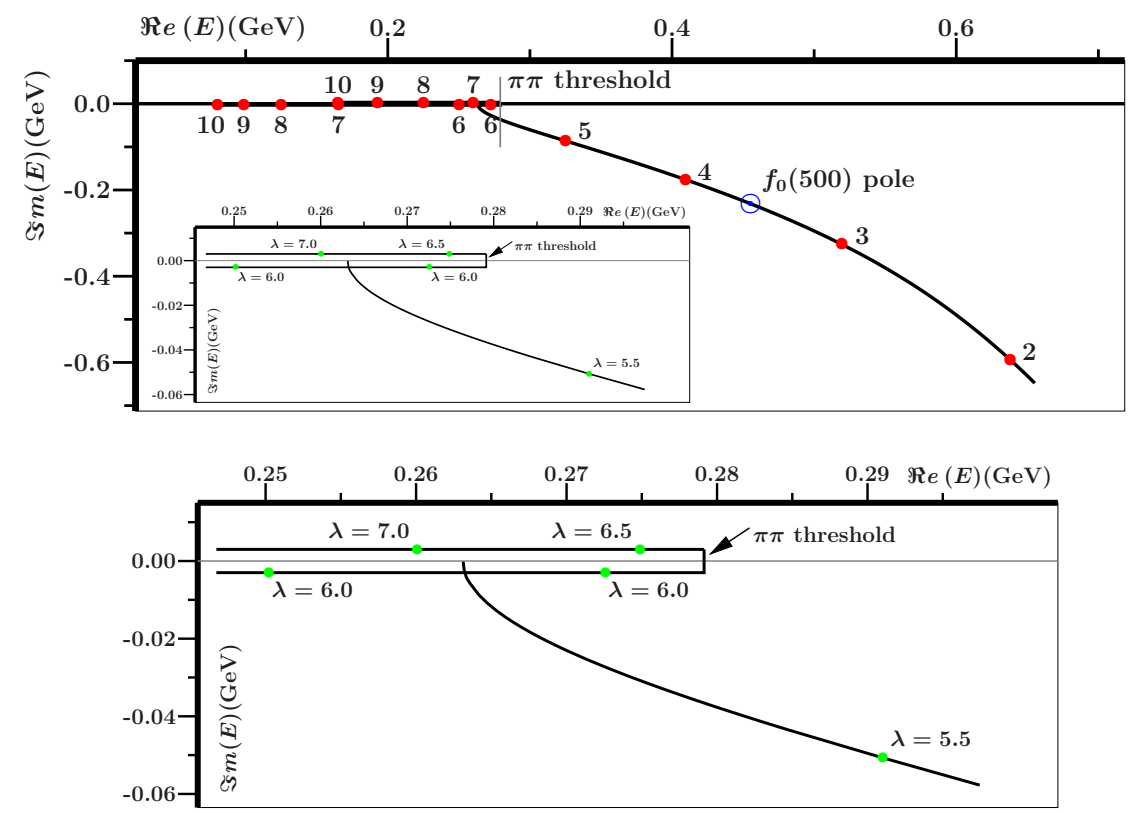

Figure 2. $f_{0}(500)$ pole trajectory as a function of $\lambda$. The open circle corresponds to the fitted $\lambda$ value. The inset (enlarged underneath) shows details of (virtual) bound states, for clarity depicted slightly (below) above the real axis. Also see text.

$a_{0}(1017-i 39.6)$ (second sheet), $a_{0}(1341-i 285)$

Poles on the second Riemann sheet are defined here as quasi-bound states with respect to the $K \bar{K}$ threshold, i.e., with positive imaginary part in the corresponding relative momentum. All these scalar-meson pole positions are very reasonable, especially for the lowest states. ${ }^{6}$

In order to better understand the nature of these resonances, we now show how they move in the complex-energy plane as a function of the overall coupling $\lambda$, as displayed in Figs. 2-4. Note that in Figs. 3 and 4 solid lines represent trajectories on what we call "natural" Riemann sheets, where the imaginary part of the relative momentum with respect to each threshold is positive or negative, according as the pole lies below or above that threshold, respectively. All other (parts of) trajectories are drawn as dashed lines in those figures. In Fig. 2 the trajectory of the $f_{0}(500)$ pole is depicted, with the inset displaying its behaviour below the $\pi \pi$ threshold. We see the pole remaining complex below threshold, as it should ${ }^{26}$ for $S$ - 

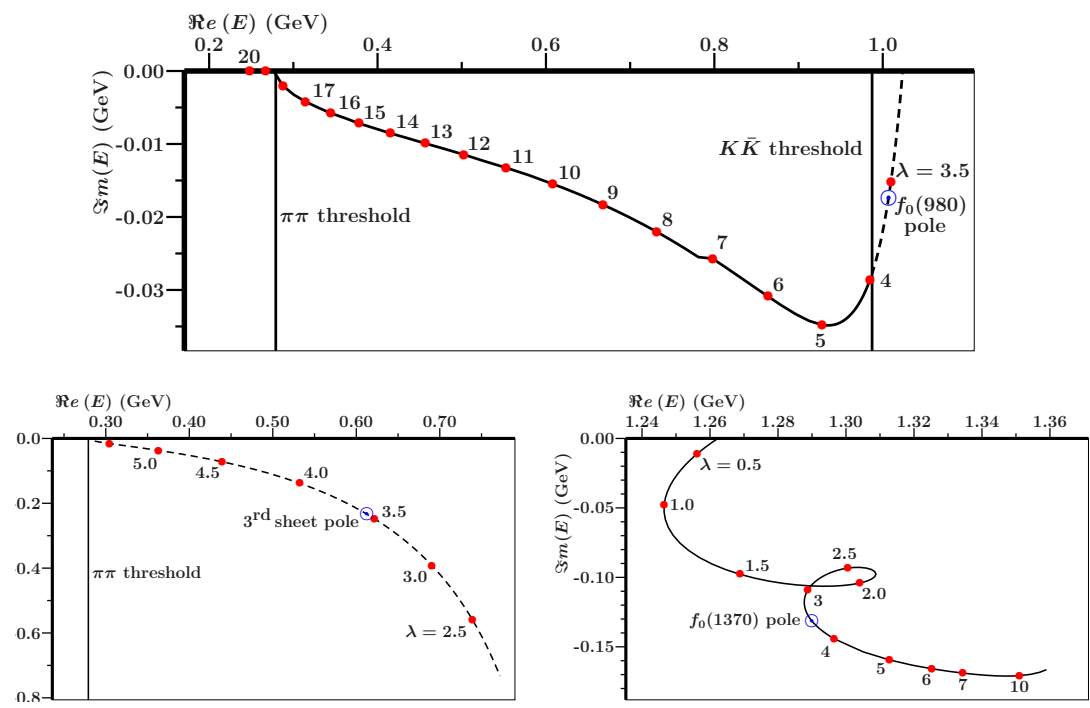

Figure 3. $f_{0}(980)$ pole trajectories as a function of $\lambda$ on second sheet (upper) and third sheet (lower left); $f_{0}(1370)$ trajectory (lower right). Also see text.

waves, and then turning into two virtual bound states, one of which moves upwards again and then becomes a true bound state. For clarity, in the inset the virtual bound states are shown somewhat below the real axis and the bound state above the axis. In the limit of small coupling, the pole moves deep into the complex plane, as a manifestation of its dynamical and not intrinsic nature. Next we inspect the $f_{0}(980)$ and $f_{0}(1370)$ trajectories in Fig. 3. Note that the pole representing $f_{0}(980)$ ends up as a secondsheet pole above the $K \bar{K}$ threshold, but is still very close to the physical region. Also, this resonance cannot be directly linked to any intrinsic $q \bar{q}$ state either, nor can the corresponding third-sheet pole, so both must be considered dynamical. Only the $f_{0}(1370)$ pole is clearly intrinsic, though with a highly non-perturbative and non-linear behaviour. The final set of trajectories concern the isovectors, depicted in Fig. 4. The dominant $a_{0}(980)$ pole lies again on the second sheet and above the $K \bar{K}$ threshold. However, this time there is also a third-sheet pole not very far from the physical region. Both $a_{0}(980)$ poles are clearly dynamical. The surprise here is that the $a_{0}(1450)$ resonance also shows up as a dynamical pole, though with a too large imaginary part. ${ }^{6}$ On the other hand, the pole of the bare $a_{0}(1450)$ state moves very far downwards into a non-physical region and so will have hardly any influence on the amplitudes for the fitted value 

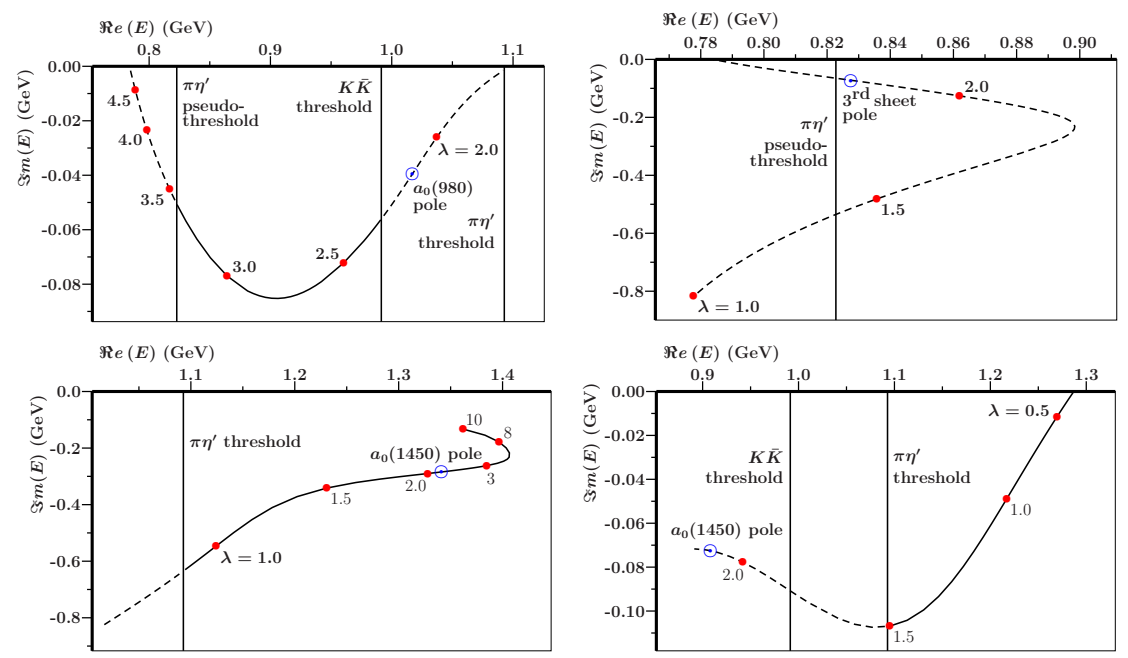

Figure 4. $a_{0}(980)$ pole trajectories as a function of $\lambda$ on second sheet (upper left) and third sheet (upper right); $a_{0}(1450)$ pole trajectories: dynamical state (lower left) and intrinsic state (lower right). Also see text.

of $\lambda$.

Summarising our findings above, it should be clear by now that the light scalar mesons are different from the other mesons in the sense that no direct connection to quark-antiquark states in the discrete confinement spectrum is possible. Their dynamical nature as $q \bar{q}$ states with large MM components has also been confirmed ${ }^{27}$ in recent years through fully unquenched lattice calculations employing both $q \bar{q}$ and MM interpolators. In the present model, also the $a_{0}(1450)$ resonance turns out to be of a dynamical nature. However, some caution is due as no scattering data in that energy region are available and so we were forced to fit the model parameters only with the $a_{0}(980)$ line shape. Moreover, for some resonances to be considered dynamical or intrinsic may hinge upon model specifics or minor changes of parameters, as e.g. in the case of $\chi_{c 1}(3872) .6,28,29$

\section{Evidence of a very light boson with a mass of $38 \mathrm{MeV}$}

The light scalar mesons described above manifest a very strong breaking of $S U(3)_{\text {flavour }}$ symmetry, in particular $f_{0}(980)$ and $a_{0}(980)$, which are almost degenerate in mass yet have very different (dominant) quark contents. However, they do not appear to correspond to the "novel" mesons with negative kinetic energy as proposed ${ }^{3}$ by Gribov and collaborators. This leaves 


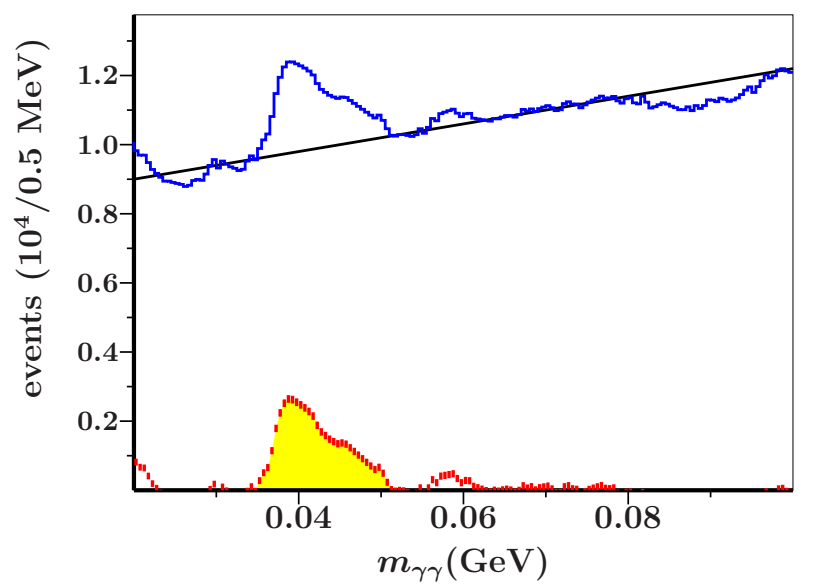

Figure 5. Top: signal in $\gamma \gamma$ data, ${ }^{31}$ with maximum around $39 \mathrm{MeV}$. Bottom: the structure remaining after background subtraction and containing about 46000 events.

open the question whether observable mesonic states from a condensate of light quarks may exist. For that matter, in Ref. ${ }^{12}$ we proposed the existence of a very light scalar boson with a mass of about $38 \mathrm{MeV}$, which we called $E(38)$. The original motivation was a decades-old geometric model ${ }^{30}$ of quark-gluon confinement based on an anti-De-Sitter metric, in which a light scalar field played a fundamental role, but only in Ref. ${ }^{12}$ we noticed first indirect experimental signals of such a boson. These amounted to small oscillations in several production data, as well as a clear and unexplained asymmetry in bottomonium decays (see Refs. ${ }^{12,32}$ for details and further theoretical support). A much more direct indication we reported in Ref. ${ }^{13}$, namely a very pronounced $\gamma \gamma$ peak in data published ${ }^{31}$ by the COMPASS collaboration, extracted by us from those data and also shown here in Fig. 5. However, this interpretation of the data was contested ${ }^{33}$ by COMPASS, followed again by our rebuttal ${ }^{34}$ of their arguments. Nevertheless, more recently an experimental group at the Joint Institute for Nuclear Research in Dubna observed ${ }^{11}$ direct $\gamma \gamma$ signals at $38 \mathrm{MeV}$ in proton and deuteron scattering off carbon and copper nuclei, though with insufficient statistics to be considered an indisputable observation.

\section{Conclusions and outlook}

As in previous published work, we have shown in this paper that the light scalar mesons can be modelled as $q \bar{q}$ states without exotic configurations, 
provided that their coupling to $S$-wave MM channels is accounted for in a unitary framework. Thus, they emerge as a nonet of dynamical resonances, which nevertheless owe their existence to bare $P$-wave $q \bar{q}$ states above $1 \mathrm{GeV}$. The latter ones also become resonances upon unitarisation, either as shifted intrinsic states or again as dynamical ones. As for $f_{0}(980)$ and $a_{0}(980)$, they end up as relatively narrow resonances close to and strongly attracted by the $K \bar{K}$ threshold, also because their lowest decay modes are suppressed. In the $f_{0}(980)$ case, this happens because of the mostly $s \bar{s}$ quark content of this state, which component does not couple to $\pi \pi$. For $a_{0}(980)$, the coupling to $\pi \eta$ is reduced because of the large pseudoscalar mixing angle and is further suppressed by the Adler zero ${ }^{21}$ just below that threshold.

In future work on the scalar mesons, we plan to generalise Eqs. $(2-5)$ so as to allow for different decay radii in all situations and will also consider alternative vertex functions. Moreover, the empirical procedure to deal with complex asymptotic masses in the $S$-matrix while preserving unitarity, as developed in Ref. ${ }^{28}$, will be employed to better deal with multiparticle decays. This may improve the phase-shift behaviour above $1 \mathrm{GeV}$.

To conclude, we stress the enormous importance of experimentally confirming the $E(38)$ boson, which could have far-reaching consequences for QCD and would also be a further tribute to Gribov's work on confinement.

\section{References}

1. V. Gribov, Eur. Phys. J. C10, 71 (1999) [arXiv:hep-ph/9807224]; 91 (1999) [arXiv:hep-ph/9902279].

2. K. Hikasa et al. [Particle Data Group], Phys. Rev. D45, S1 (1992) [Erratum: Phys. Rev. D46, 5210 (1992)].

3. F. E. Close, Y. L. Dokshitzer, V. N. Gribov, V. A. Khoze, and M. G. Ryskin, Phys. Lett. B319, 291 (1993).

4. G. Rupp and E. van Beveren Acta Phys. Polon. B Proc. Supp. 11, 455 (2018) [arXiv:1806.00364 [hep-ph]].

5. R. L. Jaffe, Phys. Rev. D15, 267 (1977); 281 (1977).

6. P. A. Zyla et al. [Particle Data Group], Prog. Theor. Exp. Phys. 2020, $083 \mathrm{C} 01$ (2020).

7. E. van Beveren, T. A. Rijken, K. Metzger, C. Dullemond, G. Rupp, and J. E. Ribeiro, Z. Phys. C30, 615 (1986) [arXiv:0710.4067 [hep-ph]].

8. Z. Y. Zhou and Z. Xiao, arXiv:2008.02684 [hep-ph], arXiv:2008.08002 [hep$\mathrm{ph}$.

9. G. Rupp, S. Coito, and E. van Beveren, Acta Phys. Polon. B Proc. Supp. 2, 437 (2009) [arXiv:0905.3308 [hep-ph]].

10. E. van Beveren and G. Rupp, Int. J. Theor. Phys. Group Theor. Nonlin. 
Opt. 11, 179 (2006) [arXiv:hep-ph/0304105].

11. K. Abraamyan, C. Austin, M. Baznat, K. Gudima, M. Kozhin, S. Reznikov, and A. Sorin, EPJ Web Conf. 204, 08004 (2019).

12. E. van Beveren and G. Rupp, arXiv:1102.1863 [hep-ph];

13. E. van Beveren and G. Rupp, arXiv:1202.1739 [hep-ph].

14. E. van Beveren and G. Rupp, arXiv:2012.03693 [hep-ph], to be published.

15. E. van Beveren, C. Dullemond, and G. Rupp, Phys. Rev. D21, 772 (1980) [Erratum: Phys. Rev. D22, 787 (1980)].

16. E. van Beveren, G. Rupp, T. A. Rijken, and C. Dullemond, Phys. Rev. D27, 1527 (1983).

17. M. Aguilar-Benitez et al. [Particle Data Group], Phys. Lett. B170, 1 (1986).

18. E. van Beveren and G. Rupp, Eur. Phys. J. C22, 493 (2001) [arXiv:hepex/0106077].

19. D. E. Groom et al. [Particle Data Group], Eur. Phys. J. C15, 1 (2000).

20. E. van Beveren and G. Rupp, Phys. Rev. Lett. 91, 012003 (2003) [arXiv:hepph/0305035].

21. E. van Beveren, D. V. Bugg, F. Kleefeld, and G. Rupp, Phys. Lett. B641, 265 (2006) [arXiv:hep-ph/0606022].

22. E. van Beveren and G. Rupp, Annals Phys. 324, 1620 (2009) [arXiv:0809.1149 [hep-ph]].

23. S. Coito, G. Rupp, and E. van Beveren, Phys. Rev. D80, 094011 (2009) [arXiv:0909.0051 [hep-ph]].

24. E. van Beveren, Z. Phys. C17, 135 (1983) [arXiv:hep-ph/0602248].

25. M. D. Scadron, G. Rupp, and R. Delbourgo, Fortsch. Phys. 61, 994 (2013) [arXiv:1309.5041 [hep-ph]].

26. J. R. Taylor, Scattering Theory: The Quantum Theory of Nonrelativistic Collisions, John Wiley \& Sons, Inc., New York, London, Sydney, Toronto, 1972, pp. 477, ISBN 0-471-84900-6.

27. J. J. Dudek et al. [Hadron Spectrum Collaboration], Phys. Rev. D93, 094506 (2016) [arXiv:1602.05122 [hep-ph]]; R. A. Briceno, J. J. Dudek, R. G. Edwards, and D. J. Wilson, Phys. Rev. Lett. 118, 022002 (2017) [arXiv:1607.05900 [hep-ph]]; Phys. Rev. D97, 054513 (2018) [arXiv:1708.06667 [hep-lat]]; D. J. Wilson, R. A. Briceno, J. J. Dudek, R. G. Edwards, and C. E. Thomas, Phys. Rev. Lett. 123, 042002 (2019) [arXiv:1904.03188 [hep-lat]].

28. S. Coito, G. Rupp, and E. van Beveren, Eur. Phys. J. C71, 1762 (2011) [arXiv:1008.5100 [hep-ph]].

29. S. Coito, G. Rupp, and E. van Beveren, Eur. Phys. J. C73, 2351 (2013) [arXiv:1212.0648 [hep-ph]].

30. C. Dullemond, T. A. Rijken, and E. van Beveren, Nuovo Cim. A80, 401 (1984).

31. T. Schluter [COMPASS Collaboration], eConf C110613, 83 (2011) [arXiv:1108.6191 [hep-ex]].

32. E. van Beveren and G. Rupp, arXiv:2005.08559 [hep-ph].

33. J. Bernhard et al. [COMPASS Collaboration], arXiv:1204.2349 [hep-ex].

34. E. van Beveren and G. Rupp, arXiv:1204.3287 [hep-ph]. 